\title{
Robust manipulation of electron spin coherence in an ensemble of singly charged quantum dots
}

\author{
A. Greilich, M. Wiemann, F. G. G. Hernandez ${ }^{\dagger}$, D. R. Yakovlev $§$, I. A. Yugova ${ }^{\ddagger}$, and M. Bayer \\ Experimentelle Physik II, Universität Dortmund, D-44221 Dortmund, Germany \\ A. Shabaev* and Al. L. Efros \\ Naval Research Laboratory, Washington, DC 20375, USA \\ D. Reuter and A. D. Wieck \\ Angewandte Festkörperphysik, Ruhr-Universität Bochum, D-44780 Bochum, Germany
}

(Dated: November 4, 2018, robustcontrol-03-27-07-fin.tex)

\begin{abstract}
Using the recently reported mode locking effect [1] we demonstrate a highly robust control of electron spin coherence in an ensemble of (In, Ga)As quantum dots during the single spin coherence time. The spin precession in a transverse magnetic field can be fully controlled up to $25 \mathrm{~K}$ by the parameters of the exciting pulsed laser protocol such as the pulse train sequence, leading to adjustable quantum beat bursts in Faraday rotation. Flipping of the electron spin precession phase was demonstrated by inverting the polarization within a pulse doublet sequence.

PACS numbers: 72.25.Dc, 72.25.Rb, 78.47.+p, 78.55.Cr
\end{abstract}

The spin of an electron in a quantum dot (QD) is an attractive quantum bit candidate [2, 3, 4, 5] due to its favorable coherence properties $[1,6], 7,8]$. As the interaction strength is rather small for direct spin manipulation, the idea to swap spin into charge has been furbished 6, 9, 10]. For example, the electron may be converted into a charged exciton by optical injection of an electronhole pair [10], depending on the residual electron's spin orientation, leading to distinctive polarization selection rules.

The fundamental quantity regarding spin coherence is the transverse relaxation time $T_{2}$. In a $\mathrm{QD}$ ensemble, this time is masked by dephasing, mostly caused by dotto-dot variations of the spin dynamics. The dephasing time does not exceed $10 \mathrm{~ns}$, much shorter than $T_{2}$. This leads to the general believe that manipulations ought to

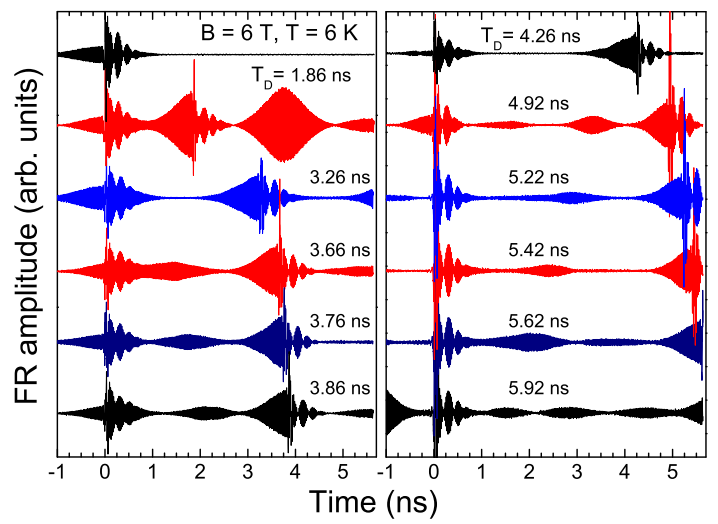

FIG. 1: Faraday rotation traces measured as function of delay between probe and first pump pulse at time zero. A second pump pulse was applied, delayed relative to the first one by $T_{D}$, indicated at each trace. The top left trace gives the FR without second pump. be performed on a single spin. Measurement of a single electron spin polarization, however, also results in dephasing due to temporal sampling of varying nuclear spin configurations [11, 12], as statistically significant measurements on a single QD may require multiple repetition of the experiment. The dephasing can be overcome by spin-echo techniques, which give a single electron spin coherence time on the scale of micro-seconds [8]. This long coherence time derived by spin-echo is result of a refocusing of the electron spin and possibly the nuclear spin configuration [11], and it is viewed as an upper bound on the free-induction decay of spin coherence [11, 13].

Recently, however, we have shown that mode locking of electron spin coherence allows one to overcome the ensemble dephasing [14] and to measure the single electron spin relaxation time $T_{2}$ without applying spin-echo refocusing [1]. For monitoring the coherence, pump-probe Faraday rotation (FR) measurements [15] on a QD ensemble were used: after optical alignment of the spins normal to an external magnetic field the electron spins precess about this field. Due to precession frequency variations the ensemble phase coherence is quickly lost. However, a periodic train of circularly polarized pulses emitted by a mode-locked laser synchronizes those spin precession modes, for which the precession frequency is a multiple of the laser repetition rate. This synchronization leads to constructive interference (CI) of these modes in the ensemble spin polarization before arrival of each pump pulse (see Fig. 1, upper left trace). The limit for spin mode locking is set by the single electron spin coherence time which can last up to a few microseconds [1] reaching the low bound on echo-like decays [16].

Here we develop a detailed understanding of the degree of control which can be reached for the electron spin coherence in an ensemble of singly charged QDs by exploiting the mode locking. For this purpose trains of excitation pump pulse doublets were designed to vary 
the phase synchronization condition (PSC) for electron spin precession frequencies. The PSC selects a QD subset, whose contribution to the ensemble spin polarization shows a well controlled phase recovery. Variation of the pulse separation results in tunable patterns of quantum oscillation bursts in time-resolved FR, in good agreement with our calculation, which rely on a newly developed theoretical model. This tailoring of electron spin coherence is very robust, as the spin mode locking is stable up to $25 \mathrm{~K}$. For higher temperatures the coherence amplitude decreases due to phonon-assisted scattering of holes during the laser pulse excitation by which the spin coherence is created.

The studied self-assembled (In,Ga)As/GaAs QDs were fabricated by molecular beam epitaxy on a (001)-oriented GaAs substrate. The sample contains 20 QD layers with a layer dot density of about $10^{10} \mathrm{~cm}^{-2}$, separated by 60 nm wide barriers [17]. For average occupation by a single electron per dot, the structures were $n$-modulation doped $20 \mathrm{~nm}$ below each layer with a Si-dopant density matching roughly the dot density. The sample was held in the insert of an optical magneto-cryostat, allowing temperature variation from $T=6$ to $50 \mathrm{~K}$.

FR with picosecond time resolution was used for studying the spin dynamics: Thereby spin polarization along the growth direction ( $z$-axis) is generated by a circularly polarized pump pulse hitting the sample along $z$, and its precession in a transverse magnetic field $B \leq 7 \mathrm{~T}$ along the $x$-axis is tested by the rotation of the linear polarization of a probe pulse. For optical excitation, a Tisapphire laser was used emitting pulses with a duration of $\sim 1.5 \mathrm{ps}$ (full width at half maximum of $\sim 1 \mathrm{meV}$ ) at $75.6 \mathrm{MHz}$ repetition rate (corresponding to a repetition period $T_{R}=13.2 \mathrm{~ns}$ ). The laser energy was tuned into resonance with the QD ground state transition and the laser pulses were split into pump and probe. The pump beam was split further into two pulses with variable delay $T_{D}$ in between. The circular polarization of the two pumps could be controlled individually. For detecting the rotation angle of the probe beam linear polarization, a homodyne technique was used.

Figure 1 shows FR traces excited by the two-pulse train with a repetition period $T_{R}=13.2 \mathrm{~ns}$, in which both pulses have the same intensity and polarization, and the delay between these pulses $T_{D}$ was varied between $\sim T_{R} / 7$ and $\sim T_{R} / 2$. The FR pattern varies strongly for the case when the delay time $T_{D}$ is commensurate with the repetition period $T_{R}: T_{D}=T_{R} / i$ with $i=2,3, \ldots$, and for the case $T_{D} \neq T_{R} / i$. For commensurability $T_{D}=T_{R} / i$, the FR signal shows strong periodic bursts of quantum oscillations only at times equal to multiples of $T_{D}$, as seen in the left panel of Fig. 1 for $T_{D}=1.86 \mathrm{~ns} \approx T_{R} / 7$. Commensurability is also given to a good approximation for delays $T_{D}=T_{R} / 4 \approx 3.26 \mathrm{~ns}$ and $T_{D}=T_{R} / 3 \approx 4.26 \mathrm{~ns}$.

For incommensurability of $T_{D}$ and $T_{R}, T_{D} \neq T_{R} / i$, the FR signal shows bursts of quantum oscillations between the two pulses of each pump doublet, in addition to the bursts outside of the doublet. For example, one can see a single burst in the mid between the pumps for $T_{D}=3.76$ and $5.22 \mathrm{~ns}$. Two bursts, each equidistant from the closest pump and also equidistant from one another, appear at $T_{D}=4.92$ and $5.62 \mathrm{~ns}$. Three equidistant bursts occur at $T_{D}=5.92 \mathrm{~ns}$. Note also that the FR amplitude before the second pump arrival is always significantly larger than before the first pump for any $T_{D}$.

Although the time dependencies of the FR signal look very different for commensurate and incommensurate $T_{D}$ and $T_{R}$, in both cases they can be fully controlled by designing the synchronization of electron spin precession modes in order to reach $\mathrm{CI}$ of their contributions to the FR signal [1]. A train of circularly polarized pump pulse singlets synchronizes those spin precessions for which the precession frequency satisfies the PSC [1, 18]: $\omega_{e}=2 \pi N / T_{R}$. Then the electron spin undergoes an integer number, $N \gg 1$, of full $2 \pi$ rotations in the interval $T_{R}$ between the pump pulses.

For a train of pump pulse doublets the PSC has to be extended to account for the intervals $T_{D}$ and $T_{R}-T_{D}$ in the laser excitation protocol

$$
\omega_{e}=2 \pi N K / T_{D}=2 \pi N L /\left(T_{R}-T_{D}\right),
$$

where $K$ and $L$ are integers. On first glance this condition imposes severe limitations on the $T_{D}$ values, for which synchronization is obtained:

$$
T_{D}=[K /(K+L)] T_{R},
$$

which for $T_{D}<T_{R} / 2$ leads to $K<L$. When Eq. (2) is satisfied, the contribution of synchronized precession modes to the average electron spin polarization $\bar{S}_{z}(t)$ is proportional to $-0.5 \cos \left[N\left(2 \pi K t / T_{D}\right)\right]$. Summing over all relevant oscillations leads to CI of their contributions with a period $T_{D} / K$ in time [1]. The rest of QDs does not contribute to $\bar{S}_{z}(t)$ at times longer than the ensemble dephasing time. The PSC Eq. (11) explains the position of all bursts in the FR signal for commensurate and incommensurate ratios of $T_{D}$ and $T_{R}$. For commensurability, $K \equiv 1$ and $T_{D}=T_{R} /(1+L)$ according to Eq. (2). In this case CIs should occur with period $T_{D}$ as seen in Fig. 1 for $T_{D}=1.86$ ns $(L=6)$.

For incommensurability of $T_{D}$ and $T_{R}$ the number of FR bursts between the two pulses within a pump doublet and the delays at which they appear can be tailored. There should be just one burst between the pulses, when $K \equiv 2$, because then the CI must have a period $T_{D} / 2$. A single burst is seen in Fig. 1 for $T_{D}=3.76$ and $5.22 \mathrm{~ns}$. The corresponding ratios $T_{D} / T_{R}$ are 0.285 and 0.395 , respectively. At the same time Eq.(2) gives a ratio $T_{D} / T_{R}=2 /(L+2)$, which is equal to 0.285 and 0.4 for $L=5$ and 3 , respectively, in good accord with experiment.

Next, two FR bursts are seen for $T_{D}=4.92$ and $5.62 \mathrm{~ns}$, corresponding to $T_{D} / T_{R} \approx 0.372$ and 0.426 . The corresponding CI period $T_{D} / 3$ is reached for $K \equiv 3$. Then from Eq.(2) $T_{D} / T_{R}=3 /(L+3)$, giving 0.375 

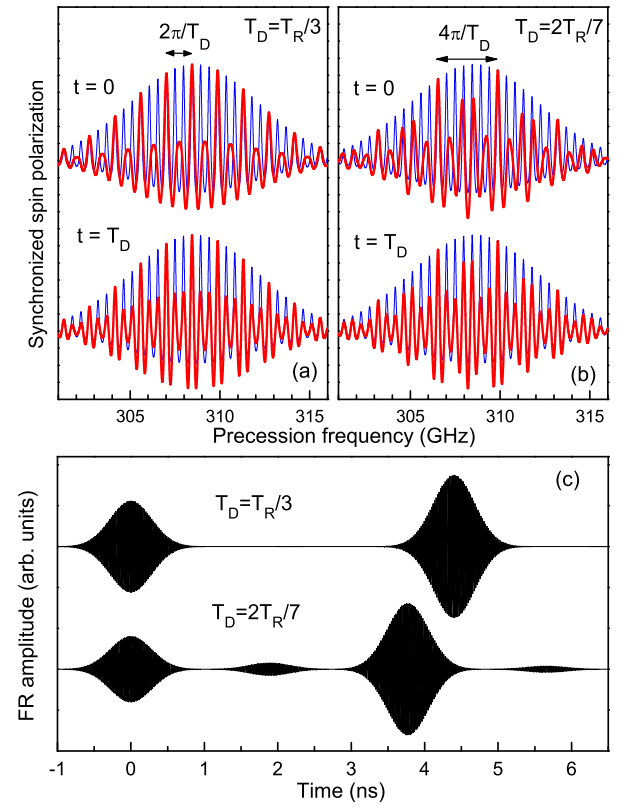

FIG. 2: (a,b): Spectra of electron spin precession modes, $-\bar{S}_{z}(t)$, which are phase synchronized by the two-pulse train calculated for $T_{D}=T_{R} / 3$ and $T_{D}=2 T_{R} / 7$ at the moments of first $(t=0)$ and second $\left(t=T_{D}\right)$ pulse arrival (red). Single-pump spectra are shown in blue. (c): FR traces calculated for two ratios of $T_{D} / T_{R}$. Laser pulse area $\Theta=\pi$. $T_{R}=13.2$ ns. Electron $g$-factor $\left|g_{e}\right|=0.57$ and its dispersion $\Delta g=0.005 . B=6 \mathrm{~T}$.

and 0.429 for $L=5$ and 4 , respectively. Finally, the FR signal with $T_{D}=5.92 \mathrm{~ns}\left(T_{D} / T_{R} \approx 0.448\right)$ shows three FR bursts between the two pumps. The CI period $T_{D} / 4$ is obtained for $K \equiv 4$, for which Eq.(2) gives $T_{D} / T_{R}=4 /(L+4) \approx 0.444$ with $L=5$. Obviously good general agreement between experiment and theory is established, highlighting the high flexibility of the laser protocol. In turn, this understanding can be used to induce FR bursts at wanted delays $T_{D} / K$, so that at these times further coherent manipulation of all electron spins involved in the burst is facilitated.

However, the question arises how accurate condition Eq. (22) for the $T_{D} / T_{R}$ ratio must be fulfilled to reach phase synchronization. Formally, one can find for any arbitrary $T_{D} / T_{R}$ large $K$ and $L$ values such that Eq. (2) is satisfied with high accuracy. But the above analysis shows, that only the smallest of all available $L$ leads to PSC matching. Experimentally, the facilities to address this point are limited, as the largest $T_{D}$ for which FR signal can be measured are delays around 5 ns between the two pumps. For larger delays the FR bursts shift out of the scanning range. For short $T_{D}$, on the other hand, the bursts are overlapping with the FR signal from the pump pulses.

To answer this question, we have modeled the FR signal for commensurate and incommensurate ratios of $T_{D}$ and $T_{R}$. Figure 2 shows the results together with spec- tra of synchronized spin precession modes (SSPM) at the moment of the first and second pump pulse arrival. The SSPM were calculated similar those induced by a single pulse train [1]. Figure 2(a) gives the SSPM for commensurate $T_{D}=T_{R} / 3$ superimposed on the SSPM created by a single pulse train with the same $T_{R}$. Panel (c) shows the FR signal created by such a two pulse train. The SSPM for the considered strong excitation are considerably broadened and contain modes for which $\omega_{e}=2 \pi M / T_{D}=2 \pi 3 M / T_{R}$ with integer $M$, which coincide with each third mode created by a single pulse train. However, the SSPM given by $\omega_{e}=2 \pi N / T_{R}$, which do not satisfy the PSC for a two pulse train, are not completely suppressed, because the train synchronizes the electron spin precession in some frequency range around the PSC. One sees also, that at $t=0$ the two pulse train leads to a significant alignment of electron spins opposite to the direction of spins satisfying the PSC. This "negative" alignment decreases the CI magnitude and therefore the FR signal before the first pulse arrival, and is also responsible for a significantly larger magnitude of the FR signal before the second pulse arrival [see Figs. 1 and 2 (c)].

For incommensurate ratios of $T_{D}$ and $T_{R}$ the SSPM become much more complex. Still we are able to recover the modes which satisfy the PSC at the pulse arrival times. In Fig. $2(\mathrm{~b})$ we show the SSPM at $t=0$ and $t=$ $T_{D}$ for $T_{D}=2 T_{R} / 7(K=2, L=5)$, where the arrows indicate the frequencies which satisfy the PSC for the two pulse train. Only a small number of such modes fall within the average distribution of electron spin precession modes, because the distance between the PSC modes is proportional to $2 \pi K / T_{D}=2 \pi(K+L) / T_{R}$. The diluted spectra of PSC modes for incommensurability decrease the magnitude of the FR bursts between the pump pulses, in accord with experiment. This shows, that although any ratio of $T_{D} / T_{R}$ can be satisfied by large $K$ and $L$, the FR signal between the pulses should be negligibly small in this case. Consequently, not any ratio of $T_{D} / T_{R}$ leads to pronounced FR bursts.

To obtain further insight into the tailoring of electron spin coherence, which can be reached by a two-pulse train, we have turned from co- to counter-circularly polarized pumps. The delay between pumps $T_{D}$ was fixed at $T_{R} / 6 \approx 2.2 \mathrm{~ns}$. The time dependencies of the corresponding FR signals are similar, as shown in Fig. 3. Besides the two FR bursts directly connected to the pump pulses, one sees a burst +1 due to CI of spin synchronized modes. The insets in Fig. 3 (a) show closeups of the different FR bursts. The sign, $\kappa$, of the FR amplitude for the counter-circular configuration undergoes $2 T_{D}$-periodic changes in time relative to the co-circular case, as seen in Fig. 3 (b), which demonstrates optical switching of the electron spin precession phase by $\pi$ in an ensemble of QDs.

The observed effect of sign reversal is well described by our model. Let us consider first a two-pulse train with delay time $T_{D}=T_{R} / 2$ for which the two pumps 


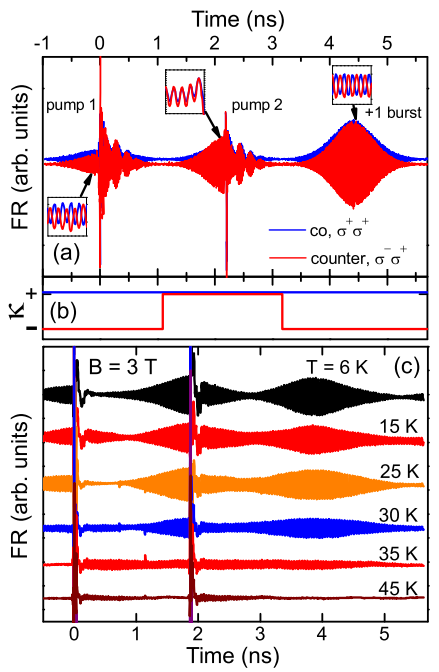

FIG. 3: (a): Faraday rotation traces in the co-circularly (blue traces) or counter-circularly (red traces) polarized two pump pulse experiments measured for $T_{D}=2.2 \mathrm{~ns}$ and $B=6 \mathrm{~T}$. Insets give close-ups showing the relative sign, $\kappa$, of the FR amplitude between the two traces. $\kappa$ is plotted in (b) vs time. (c): Effect of temperature on the FR amplitude in twopump-pulse experiment. $T_{D}=1.88 \mathrm{~ns}$.

are counter-circularly polarized. In this case an electron spin can be synchronized only if at the moment of pulse arrival it has an orientation opposite to the orientation at the previous pulse. This leads to the PSC $\omega_{e}=$ $2 \pi(N+1 / 2) / T_{D}$. The contribution of such precession modes to the electron spin polarization is proportional to $\left.\cos \left[2 \pi(N+1 / 2) t / T_{D}\right)\right]=\cos \left(2 \pi N t / T_{D}\right) \cos \left(\pi t / T_{D}\right)-$ $\sin \left(2 \pi N t / T_{D}\right) \sin \left(\pi t / T_{D}\right)$. Summing these contributions, only the first term gives a CI, whose modulus has period $T_{D}$, while the sign of $\cos \left(\pi t / T_{D}\right)$ changes with period $2 T_{D}$. Only each third of the precession frequencies can be synchronized by a counter-circularly polarized two pulse train when the delay time is $T_{R} / 6$ as in our experiment. However, the corresponding PSC has the same dependence on $T_{D}$. The CI modulus also has period $T_{D}$ and its sign changes with period $2 T_{D}$. The relative sign of the FR amplitude for the counter- and co-circularly case, $\kappa=\operatorname{sgn}\left\{\cos \left[\pi t / T_{D}\right]\right\}$, is in accord with the experimental dependence in Fig. 3 (b).
The CIs of the electron spin contributions can be seen only as long as the coherence of the electron spins is maintained. In this respect the temperature stability of the CI is especially important. Fig. 3 (c) shows FR traces in a two-pump-pulse configuration with $T_{D}=1.88 \mathrm{~ns}$ at different temperatures. For both positive and negative delays, the FR amplitude at a fixed delay is about constant for temperatures up to $25 \mathrm{~K}$, irrespective of slight variations which might arise from changes in the phase synchronization of QD subsets. Above $30 \mathrm{~K}$ a sharp drop occurs, which can be explained by thermally activated destruction of the spin coherence.

The electron spin coherence in charged QDs is initiated by generation of a superposition of an electron and a charged exciton state by resonant pump pulses [17, 19]. The simultaneous decrease of the FR magnitude before each pump pulse and afterwards (when the CI signal is controlled by the excitation pulse) suggests that the coherence at elevated temperatures is lost already during its generation. The $30 \mathrm{~K}$ temperature threshold corresponds to an activation energy of $\sim 2.5 \mathrm{meV}$. This energy may be assigned only to the splitting between the two lowest confined hole levels, because the electron level splitting dominates the $20 \mathrm{meV}$ splitting between p- and s-shell emission in photoluminescence and is much larger than $2.5 \mathrm{meV}$. The decoherence of the hole spin results from two phonon scattering, which is thermally activated and should occur on a sub-picosecond time scale, i.e. within the laser pulse [20]. The fast decoherence of the hole spin at $T>30 \mathrm{~K}$ suppresses formation of the electron-trion superposition state. ps-pulses as used here are therefore not sufficiently short for initialization of the superposition and creation of a long-lived electron spin coherence.

In summary, we have demonstrated that the modelocking effect allows a far-reaching control of electron spin coherence in QD ensembles during the spin coherence time of microseconds [1]. Two-pulse train modelocking selects QD subsets which give a non-dephasing contribution to the ensemble spin precession. The technique shows remarkable stability with respect to temperature increase up to $25 \mathrm{~K}$, a property which is important for utilizing it in quantum information processing. The robustness of this control technique is provided by the dispersion of the spin precession frequencies in the $\mathrm{QD}$ ensemble.

Acknowledgments. This work was supported by the BMBF program nanoquit, the DARPA program QuIST, the ONR, the DFG (FOR485) and FAPESP. $\left.{ }^{\dagger}\right]$ on leave from the Instituto de Fisica Gleb Wataghin, Campinas, SP, Brazil.

[§] also at Ioffe Physico-Technical Institute, 194021, St. Petersburg, Russia.

$\left[{ }^{\ddagger}\right]$ also at Institute of Physics, St. Petersburg State University, 198504, St. Petersburg, Russia.
[* ] also at School of Computational Sciences, George Mason University, Fairfax VA 22030.

[1] A. Greilich et al., Science 313, 341 (2006).

[2] D. Loss and D. P. DiVincenzo, Phys. Rev. A 57, 120 (1998).

[3] A. Imamoglu et al., Phys. Rev. Lett. 83, 4204 (1999). 
[4] Semiconductor Spintronics and Quantum Computation, ed. by D. D. Awschalom, D. Loss, and N. Samarth (Springer-Verlag, Heidelberg 2002).

[5] S. M. Clark et al., cond-mat/0610152

[6] J. M. Elzerman et al., Nature 430, 431 (2004).

[7] M. Kroutvar et al., Nature 432, 81 (2004).

[8] J. R. Petta et al., Science 309, 2180 (2005).

[9] see, for example, R. Hanson et al., Phys. Rev. Lett. 94, 196802 (2005).

[10] see, for example, T. Calarco et al., Phys. Rev. A 68, 012310 (2003); P. Chen et al., Phys. Rev. B 69, 075320 (2004).

[11] W. Yao, R. Liu, and L. J. Sham, Phys. Rev. B 74, 195301 (2006).
[12] R. Liu, S. E. Economou, L. J. Sham, and D. G. Steel, Phys. Rev. B 75, 085322 (2007).

[13] W. A. Coish et al., Phys. Stat. Sol. (b) 243, 3658 (2006).

[14] For a general treatment on suppression of phase noise see A. G. Kofman and G. Kurizki, Phys. Rev. Lett. 93, 130406 (2004).

[15] J. M. Kikkawa and D. D. Awschalom, Science 287, 473 (2000).

[16] R. Hanson et al., cond-mat/0610433

[17] A. Greilich et al., Phys. Rev. Lett. 95, 227401 (2006).

[18] A. Shabaev et al., Phys. Rev. B 68, 201305(R) (2003).

[19] T. A. Kennedy et al., Phys. Rev. B 73, 045307 (2006).

[20] T. Takagahara, Phys. Rev. B 62, 16840 (2000). 